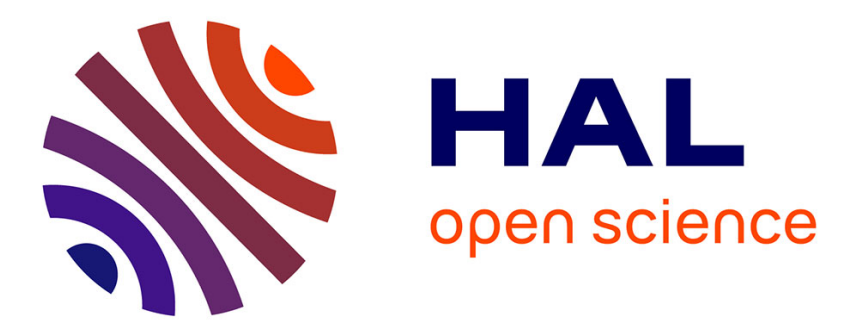

\title{
Active contour segmentation with a parametric shape prior: Link with the shape gradient
}

Eric Debreuve, Michel Barlaud, Jean-Paul Marmorat, Gilles Aubert

\section{To cite this version:}

Eric Debreuve, Michel Barlaud, Jean-Paul Marmorat, Gilles Aubert. Active contour segmentation with a parametric shape prior: Link with the shape gradient. International Conference on Image Processing (ICIP), Oct 2006, Atlanta, Georgia (GA), United States. pp.1. hal-00417711

\section{HAL Id: hal-00417711 \\ https://hal.science/hal-00417711}

Submitted on 2 Apr 2014

HAL is a multi-disciplinary open access archive for the deposit and dissemination of scientific research documents, whether they are published or not. The documents may come from teaching and research institutions in France or abroad, or from public or private research centers.
L'archive ouverte pluridisciplinaire HAL, est destinée au dépôt et à la diffusion de documents scientifiques de niveau recherche, publiés ou non, émanant des établissements d'enseignement et de recherche français ou étrangers, des laboratoires publics ou privés. 


\title{
Active contour segmentation with a parametric shape prior: link with the shape gradient
}

\author{
É. Debreuve, M. Barlaud*, J.-P. Marmorat $\dagger^{\dagger}$ G. Aubert ${ }^{\ddagger}$ \\ PREPRINT - Published in International Conference on Image Processing (ICIP) 2006
}

\begin{abstract}
Active contours are adapted to image segmentation by energy minimization. The energies often exhibit local minima, requiring regularization. Such an a priori can be expressed as a shape prior and used in two main ways: (1) a shape prior energy is combined with the segmentation energy into a trade-off between prior compliance and accuracy or (2) the segmentation energy is minimized in the space defined by a parametric shape prior. Methods (1) require the tuning of a data-dependent balance parameter and methods (1) and (2) are often dedicated to a specific prior or contour representation, with the prior and segmentation aspects often meshed together, increasing complexity. A general framework for category (2) is proposed: it is independent of the prior and contour representations and it separates the prior and segmentation aspects. It relies on the relationship shown here between the shape gradient, the prior-induced admissible contour transformations, and the segmentation energy minimization.
\end{abstract}

Keywords: Image segmentation, shape, optimization methods.

\section{Introduction}

Image or video segmentation can be formulated as an energy minimization problem. The active contour technique $[12,2,3]$ provides a convenient framework to solve this problem iteratively. Usually, there exists local minima due to noise (the energy is too sensitive) or coarseness of the models of the objects of interest (the energy is not specific enough). Therefore, regularization must be used. For instance, in order to reduce the

${ }^{*}$ É. Debreuve and M. Barlaud are with the Laboratoire I3S, UMR CNRS 6070, Université de Nice-Sophia Antipolis, France debreuve, barlaud@i3s.unice.fr

${ }^{\dagger}$ J.-P. Marmorat is with the École des Mines de Paris, C.M.A. Sophia Antipolis, France Marmorat@sophia.inria.fr

${ }^{\ddagger}$ G. Aubert is with the Laboratoire Dieudonné, UMR CNRS 6621, Université de Nice-Sophia Antipolis, France gaubert@math.unice.fr influence of noise on the solution active contour, it is classical to impose a minimal length constraint, which encourages smoothness of the contour. If the knowledge on the solution is more specific, it can be expressed in the form of a so-called shape prior. For instance, the solution must be a circle or as close to a circle as possible. There are two main approaches to imposing such a constraint (called loose and strict hereafter, respectively): (1) a shape prior energy is defined and linearly combined with the segmentation energy to form a trade-off energy between prior compliance and segmentation accuracy $[18,4,7,9]$ and (2) the segmentation energy is minimized in the space defined by a parametric shape prior constraint, either directly $[13,17]$ or using a projection strategy [5]. The advantage of the loose constraint approach is that, if the shape prior can only represent a small variety of shapes (for example, if it has been learned using a limited training set), the weight of the constraint can be reduced. Conversely, if the data is extremely noisy or incomplete, the weight of the constraint can be increased. However, this feature can also be seen as a requirement to tune a datadependent balance parameter. The strict constraint approach does not have this requirement. Nevertheless, the methods based on this approach (and also the methods based on the loose constraint approach for that matter) are classically developed for a specific contour representation (e.g., polygonal active contour [5] or level set representation [13, 17]) and/or for a specific shape prior representation (e.g., point distribution models $[5,18,7]$ or distance to a shape of reference $[4,9])$. Moreover, the shape prior and segmentation aspects are usually meshed together, making the development for complex shape priors and sophisticated segmentation energies quite unpleasant.

Here, we propose a strict constraint framework independent of the contour representation, independent of the shape prior representation, and which completely separates the shape prior aspect from the segmentation aspect, making the shape optimization part common to any shape prior. Therefore, with the same framework, shape prior constraints can range from extremely restrictive such as "the contour is a circle" to 
quite soft such as "the contour is a closed spline with $n$ control points" [10]. If the framework is independent of the contour and shape prior representations, the implementation of methods that can be developed within this framework are not. However, the specific work that has to be done is minimal and straightforward. This framework relies on the notion of shape gradient $[15,16,8,1,11]$ from the shape optimization theory and the relationship between (i) shape gradient, (ii) the admissible contour transformations defined by the shape prior constraint, and (iii) the minimization of the segmentation energy. This relationship is shown in Section 4 and results on the same image are provided for different shape priors in Section 6 .

\section{Parametric active contour and shape prior}

By parametric active contour, we do not refer to an active contour represented by a parametric curve but rather to an active contour $\Gamma$ defined by a set of parameters $p=\left\{p_{i}, i \in[1, m]\right\}$. For example, if the active contour is represented by a polygon with $n$ edges, then the parameters involved are the coordinates $\left(a_{i}, b_{i}\right)$ of each vertex $\Gamma_{i}$

$$
p=\left\{p_{j}, j \in[1,2 n]\right\}=\left\{a_{1}, b_{1}, a_{2}, b_{2} \ldots a_{n}, b_{n}\right\} .
$$

The active contour can be a parametric curve, though. For example, if it is a spline defined by $n$ control points, then the parameters involved are the coordinates $\left(a_{i}, b_{i}\right)$ of each control point $q_{i}$ and it is, by definition, a parametric curve.

This definition of parametric active contour is a way to express a shape prior. For example, it fits the combined use of a point distribution model and principal component analysis (PCA) [5, 18] or level set model and PCA $[13,17]$ since, in both cases, the active contour is expressed as the linear combination of the average shape and the principal modes of variation. Therefore, the parameters involved are the weights of the different modes

$$
\Gamma=\bar{\Gamma}+Q p
$$

where $\bar{\Gamma}$ is the average shape, $Q$ is the matrix of the first $n$ eigenvectors (sorted in descending order of their respective eigenvalues) of the covariance matrix of the deviations of the training set from the average shape, and $p$ is a weighting vector playing here the role of the $n$ parameters defining the active contour. If the active contour is represented by a level set $[13,17], \Gamma$ and $\bar{\Gamma}$ are replaced with $u$ and $\bar{u}$, the level sets representing $\Gamma$ and $\bar{\Gamma}$, respectively.

More generally, this definition of parametric active contour fits any shape prior based on shape and pose parameters.

\section{From unconstrained to con- strained segmentation}

\subsection{Segmentation as a minimization problem}

The general form of region-based energies for segmentation is

$$
E_{r}(\Omega)=\int_{\Omega} \phi(x, \Omega) \mathrm{d} x
$$

where $\Omega$ is the interior domain of the (oriented) active contour $\Gamma$ (namely, $\Gamma=\partial \Omega$ ) and $\phi$ is a function used to describe the object of interest. Ideally, it is equal to zero for all $x$ in $\Omega$ if $\Omega$ is the region of an object of interest. Otherwise it is positive. For example,

$$
\phi(x, \Omega)=(f(x)-\mu(\Omega))^{2}
$$

where $f$ is a grayscale image and $\mu(\Omega)$ is the average intensity of $f$ within $\Omega$, can be used to describe an object homogeneous in intensity. As a consequence, segmentation is expressed as a problem of energy minimization, which can be solved iteratively: an initial contour $\Gamma^{0}$ is defined and it is progressively deformed until it minimizes the energy. Each applied deformation is determined according to a notion of gradient which, particularly for region-based energies, has been studied in shape optimization and is known as the shape gradient $[15,16,8,1,11]$. Its general expression is

$$
\begin{aligned}
\mathrm{d} E_{r}(\Omega, V) & =\left.\int_{\Omega} \frac{\partial \phi(x, \Omega(\tau))}{\partial \tau}\right|_{\tau=0} \mathrm{~d} x \\
& -\int_{\Gamma} \phi(s, \Omega) V(s) \cdot N(s) \mathrm{d} s
\end{aligned}
$$

where vector $V$ is by definition a velocity defined on $\Omega, \tau$ is a deformation parameter, $s$ is the arc-length parameterization of $\Gamma$, and vector $N$ is the inward unit normal of $\Gamma$. At a given iteration, the deformation is equal to a velocity chosen so that the shape gradient is negative times a constant or optimal step. Under some conditions [11], which will be assumed to be fulfilled in the following, derivative (6) can be rewritten as

$$
\mathrm{d} E_{r}(\Omega, V)=-\int_{\Gamma} \Psi(s, \Omega) V(s) \cdot N(s) \mathrm{d} s .
$$

The general form of boundary-based energies for segmentation is

$$
E_{b}(\Omega)=\int_{\Gamma} \varphi(s) \mathrm{d} s
$$

where $\varphi$ is a function used to describe the boundary of the object of interest. Ideally, it is equal to zero for all $s$ if $\Gamma$ is the boundary of an object of interest. Otherwise it is positive. For example,

$$
\varphi(s)=\frac{1}{1+|\nabla f(\Gamma(s))|}
$$


where $\nabla f$ is the spatial gradient of $f$, can be used to describe the boundary of an object with high contrast with respect to the background. The shape gradient $[16,8]$ of $(8)$ is equal to

$$
\begin{aligned}
\mathrm{d} E_{b}(\Omega, V) & =\int_{\Gamma}\left(\frac{\partial \varphi(s)}{\partial N}-\varphi(s) \kappa(s)\right) V(s) N(s) \mathrm{d} s \\
& =\int_{\Gamma} \xi(s) V(s) N(s) \mathrm{d} s
\end{aligned}
$$

where $\kappa$ is the curvature of $\Gamma$.

Finally, the general form of energies for segmentation is a linear combination of (3) and (8), leading to a shape gradient verifying the same linear combination of (7) and (11), which results in an expression of the form of either one of them. To fix the ideas, Eq. (7) will be used in the following to refer to the general expression of the shape gradient.

\subsection{Segmentation under constraint}

Since the active contour is defined by a set of parameters, the energy can actually be rewritten as a function of $\mathbb{R}^{n}$

$$
\begin{aligned}
E(p) & =E_{r}(p)+E_{b}(p) \\
& =\int_{\Omega(p)} \phi(x, \Omega(p)) \mathrm{d} x+\int_{\Gamma(p)} \varphi(s) \mathrm{d} s .
\end{aligned}
$$

Therefore, the problem of minimizing the energy becomes classical. Starting from an initial estimate, a gradient descent method can be applied to find the minimizer. This requires computation of the derivative of the energy with respect to each parameter. As mentioned in Section 1, it can be quite complex to do it directly with a calculus of variation, depending on the shape prior and function $\phi$ in the energy. For example, if $\phi$ depends on $\Omega$ through integral terms, the complexity might lead to purposely ignore the variation of these terms with $\Omega$, only accounting for the primary variation of the energy with $\Omega[10]$. However, in Section 4, it will be shown that the derivative of the energy with respect to a parameter is given by the shape gradient of the energy evaluated for the infinitesimal deformation (otherwise called velocity) induced to the active contour by an infinitesimal change of the parameter. Intuitively, the derivative of the energy is given by a kind of chain rule between the derivative of the energy with respect to the contour (the shape gradient) and the derivative of the contour with respect to the parameter.

\section{Link between the constraint and the shape gradient}

As mentioned in Section 3.2, the gradient of energy (12) must be computed. Let us see how this gradient relates to the shape gradient. By definition, the derivative of (12) with respect to the $i^{\text {th }}$ parameter is

$$
\frac{\partial E}{\partial p_{i}} \stackrel{\text { def }}{=} \lim _{\tau \rightarrow 0} \frac{E\left(p+\tau e_{i}\right)-E(p)}{\tau}
$$

where $e_{i}$ is the $i^{\text {th }}$ element of the canonical basis of $\mathbb{R}^{n}$. This derivative involves the energies of $\Omega(p)$ and $\Omega(p+$ $\left.\tau e_{i}\right)$. The latter can be considered as a transformation of the former

$$
\Omega\left(p+\tau e_{i}\right)=T_{i}(\tau, \Omega(p)) .
$$

Note that domain $\Omega$ alone is mentioned here, $\Gamma$ being implicitly involved as a subset of $\Omega$. Therefore, the following development is valid for both integrals in (12).

Locally, transformation $T_{i}$ is defined as follows

$$
x(\tau)=T_{i}(\tau, x), x \in \Omega(p) .
$$

By definition, the velocity at $x, x \in \Omega(p)$, is

$$
\begin{aligned}
V_{i}(x) & \stackrel{\text { def }}{=} \lim _{\tau \rightarrow 0} \frac{x(\tau)-x}{\tau} \\
& =\lim _{\tau \rightarrow 0} \frac{T_{i}(\tau, x)-T_{i}(0, x)}{\tau} \\
& \stackrel{\text { def }}{=} \frac{\partial T}{\partial \tau}(\tau=0, x) .
\end{aligned}
$$

The energy of a transformed domain can be rewritten as

$$
E\left(p+\tau e_{i}\right)=E\left(\Omega\left(p+\tau e_{i}\right)\right)=E\left(\Omega(p), T_{i}, \tau\right) .
$$

With these notations, derivative (14) is equal to

$$
\frac{\partial E}{\partial p_{i}}=\lim _{\tau \rightarrow 0} \frac{E\left(\Omega(p), T_{i}, \tau\right)-E\left(\Omega(p), T_{i}, 0\right)}{\tau}
$$

which is, by definition, equal to the shape gradient $\mathrm{d} E\left(\Omega(p), T_{i}\right)$ at $\Omega(p)$ related to the domain transformation $T_{i}[15,16,8,1,11]$. Developing the shape gradient leads to an expression involving the derivative (19) of $T_{i}$. As a consequence, the shape gradient is denoted by $\mathrm{d} E\left(\Omega(p), V_{i}\right)$ instead of $\mathrm{d} E\left(\Omega(p), T_{i}\right.$ ) (see Eq. (7)). Eq. (7) only involves the restriction of $V_{i}$ to $\Gamma(p)$. It is equal to

$$
\left.V_{i}\right|_{\Gamma(p)} \stackrel{\text { def }}{=} \frac{\partial \Gamma}{\partial p_{i}} .
$$

Therefore, the derivative of (12) with respect to the $i^{\text {th }}$ parameter is equal to

$$
\frac{\partial E}{\partial p_{i}}=\mathrm{d} E\left(\Omega(p), \frac{\partial \Gamma}{\partial p_{i}}\right)
$$

i.e., it is equal to the shape gradient at $\Omega(p)$ related to the domain transformation whose restriction to $\Gamma(p)$ is equal to $\frac{\partial \Gamma}{\partial p_{i}}$. 


\section{Minimization algorithm}

Finally, the gradient of (12) with respect to $p$ is equal to (see $(23))$

$$
\nabla_{p} E=\left(\cdots \frac{\partial E}{\partial p_{i}} \cdots\right)^{\mathrm{T}}
$$

where $M^{\mathrm{T}}$ is the transpose of $M$, and a possible iterative minimization procedure is

$$
\left\{\begin{array}{l}
p^{0} \\
p^{+1}=p-\alpha \nabla_{p} E \\
\alpha=\arg \min _{a \geq 0} E\left(p-a \nabla_{p} E\right)
\end{array} .\right.
$$

The proposed framework for segmentation under a parametric shape prior constraint leads to the following algorithm

1. Define a constraint-free segmentation energy with (3) and (8)

2. Determine the general expression (7) of its shape gradient

3. Define, independently, a parametric shape prior $\Omega(p)$, or equivalently $\Gamma(p)$, with parameters $p=\left\{p_{i}, i \in[1, n]\right\}$

4. Determine the general expressions of the $n$ induced velocities $V_{i}$ equal to $\frac{\partial \Gamma}{\partial p_{i}}$

5. Initialize the constrained segmentation by choosing $p^{k}, k=0$

6. Compute the induced velocities $V_{i}^{k}$ of $\Gamma\left(p^{k}\right)$ for the $p_{i}^{k}$ 's

7. Form the gradient $\nabla_{p} E\left(p^{k}\right)$ of (12) from the $V_{i}^{k}$ 's (see $(24))$

8. Update $p^{k}$ according to (25)

9. Test if (12) kept approximately the same value these last few iterations; if it is the case, $\Gamma\left(p^{k}\right)$ is considered to be the minimizer of (12); otherwise, go back to step 6 .

Note that the shape prior is separate from the segmentation aspect and that it requires minimal effort to combine these two aspects together since it suffices to determine the induced velocities, which is trivial given the equation of $\Gamma$ as a function of $p$, and to plug them into the shape gradient expression. Changing the shape prior is only a matter of computing the new induced velocities.

\section{Experiments}

A simple, region-based energy was chosen to test the proposed method

$$
\begin{aligned}
E(p)= & \int_{\Omega(p)}|f(x)-\mu(\Omega(p))|^{2} \mathrm{~d} x \\
& +\int_{\Omega_{c}(p)}\left|f(x)-\mu\left(\Omega_{c}(p)\right)\right|^{2} \mathrm{~d} x
\end{aligned}
$$

where $f$ is a color image to be segmented (a function from $\mathbb{R}^{2}$ to $\left.\mathbb{R}^{3}\right), \mu(\Omega(p))$ is the vector of $\mathbb{R}^{3}$ of the average intensities per component of $f$ within $\Omega(p)$, and $\Omega_{c}(p)$ is the complement of $\Omega(p)$ in the image domain. The function $\phi$ used here is a color version of example (4). The combination of an integral over $\Omega(p)$ with an integral over $\Omega_{c}(p)$ is known as region competition $[14,19,6,11]$. If $\phi$ is equal to zero everywhere in the region of an object of interest, the $\Omega(p)$-energy is the same on any subset of the region. Therefore, the $\Omega(p)$-minimization process might converge to one of these subsets. Adding a description of the background (the $\Omega_{c}(p)$-energy) ensures that if a portion of the object is left outside $\Omega(p)$, the mismatch with the description of $\Omega_{c}(p)$ will tend to deform the active contour in order to include this portion. Here, the descriptions are identical, leading to a solution with a maximal separation of $\mu(\Omega(p))$ and $\mu\left(\Omega_{c}(p)\right)$ [19].

We segmented the same color image with several shape priors, ranging from restrictive to soft: the active contour was successively constrained to be a circle (parameters $p$ being the coordinates of the center and the radius), an ellipse (parameters $p$ being the coordinates of the center, the short and long axes, and the tilt angle), and a cubic B-spline with 3, 6 and 9 control points (parameters $p$ being the coordinates of the 3,6 and 9 control points, respectively). The initial contour $\Gamma\left(p^{0}\right)$ remained consistent throughout the different shape priors. First, an initial circle was chosen for the circle prior. Then, for the spline with 3 control points for example, the aforementioned initial circle was regularly sampled by 3 points and the 3 control points corresponding to the uniform cubic B-spline interpolating these points were determined and used as the initial parameters of the spline prior. The results are presented in Figs. 1 and 2.

\section{Conclusion}

The accuracy of the presented results mostly depends on (i) whether energy (26) is a convex function of $\mathbb{R}^{n}$ and, if not, (ii) whether the minimization algorithm is able to escape from local minima in order to converge to the global minimum. Concerning (i), intuitively, as the number of parameters used to describe the shape 
prior increases, energy (26) will tend to exhibit more and more local minima. Concerning (ii), the steepest descent algorithm proposed in Section 5 does not guarantee that the minimum found is global. It could be replaced by, e.g., a simulated annealing algorithm.

\section{Acknowledgments}

The image used for the experiments is a cheek cell (1000X) from the "Biology 101 - Principles of Biology" course of the Division of Biological Sciences, The University of Montana, Missoula (MT).
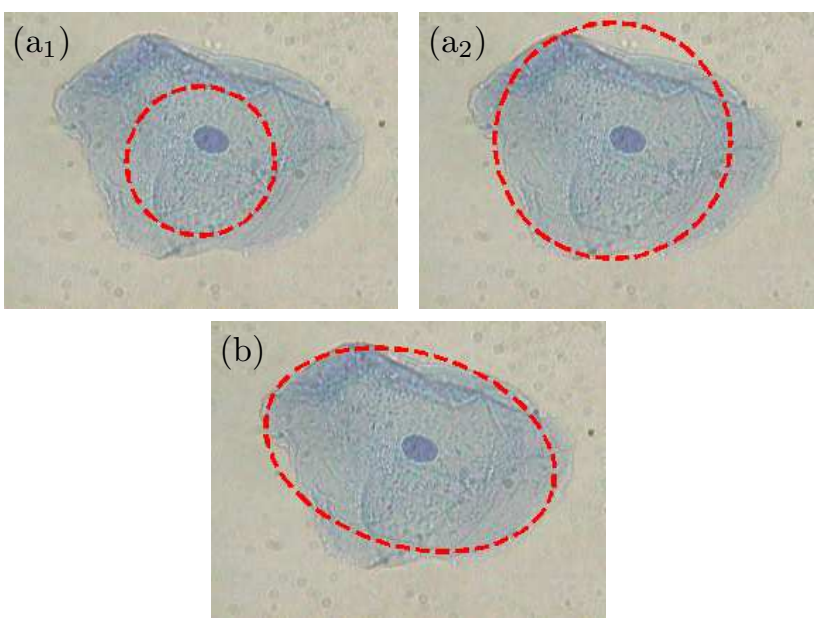

Figure 1: Circle (3 parameters) minimizing color homogeneity energy $(26)$ : $\left(a_{1}\right)$ initial estimate; $\left(a_{2}\right)$ obtained solution. (b) Ellipse ( 5 parameters) minimizing color homogeneity energy (26); the initial estimate was the same as the one used for the circle-based minimization (see $\left(a_{1}\right)$ ) although represented as an ellipse with the short and long axes equal in length.

\section{References}

[1] G. Aubert, M. Barlaud, O. Faugeras, and S. Jehan-Besson. Image segmentation using active contours: Calculus of variations or shape gradients? SIAM J. Appl. Math., 63:2128-2154, 2003.

[2] V. Caselles, F. Catté, T. Coll, and F. Dibos. A geometric model for active contours. Nümer. Math., 66:1-31, 1993.

[3] V. Caselles, R. Kimmel, and G. Sapiro. Geodesic active contours. Int. J. Comput. Vision, 22:61-79, 1997.

[4] Y. Chen, H. D. Tagare, S. Thiruvenkadam, F. Huang, D. Wilson, K. S. Gopinath, R. W. Briggs, and E. A. Geiser. Using prior shapes in
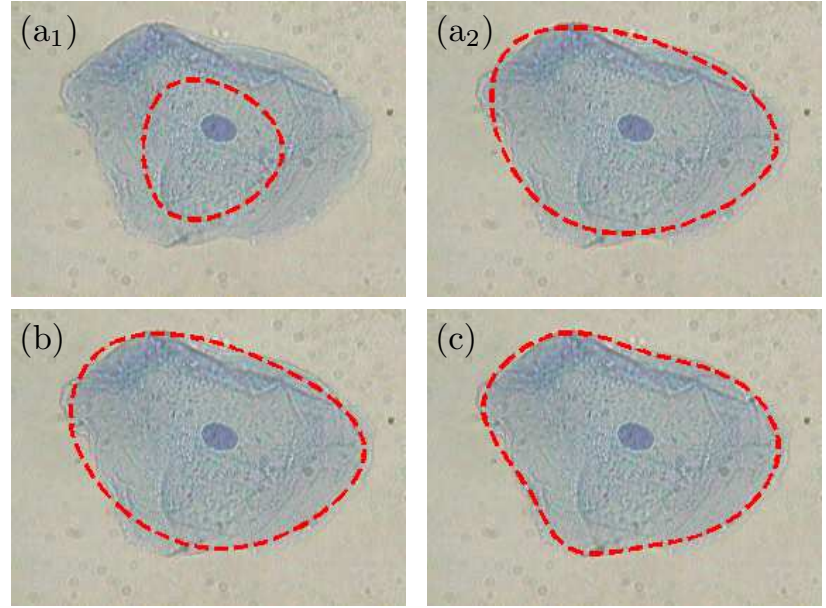

Figure 2: Splines minimizing color homogeneity energy (26): $\left(\mathrm{a}_{1}\right)$ initial spline with 3 control points $(6$ parameters) interpolating the initial circle used for the circle-based minimization (see Fig. 1); ( $\left.a_{2}\right)$ obtained spline with 3 control points; (b) spline with 6 control points (12 parameters) obtained with a spline interpolation of the aforementioned circle as the initial estimate (this interpolation is virtually indistinguishable from the circle); (c) spline with 9 control points (18 parameters) obtained with a spline interpolation of the aforementioned circle as the initial estimate (again virtually indistinguishable from the circle).

geometric active contours in a variational framework. Int. J. Comput. Vision, 50:315-328, 2002.

[5] T. F. Cootes, C. J. Taylor, D. H. Cooper, and J. Graham. Active shape models - their training and application. Comput. Vis. Image Und., 61:38$59,1995$.

[6] D. Cremers and C. Schnörr. Motion competition: Variational integration of motion segmentation and shape regularization. In $D A G M, P R$, 2002 .

[7] D. Cremers, F. Tischhäuser, J. Weickert, and C. Schnörr. Diffusion snakes: introducing statistical shape knowledge into the Mumford-Shah functional. Int. J. Comput. Vision, 50:295-313, 2002.

[8] M. C. Delfour and J.-P. Zolésio. Shapes and geometries: Analysis, differential calculus and optimization. Advances in Design and Control. Society for Industrial and Applied Mathematics, Philadelphia, 2001.

[9] M. Gastaud, M. Barlaud, and G. Aubert. Combining shape prior and statistical features for active contour segmentation. IEEE Trans. Circ. Syst. Vid., 14:726-734, 2004. 
[10] M. Jacob, T. Blu, and M. Unser. Efficient energies and algorithms for parametric snakes. IEEE Trans. Image Process., 13:1231-1244, 2004.

[11] S. Jehan-Besson, M. Barlaud, and G. Aubert. $\mathrm{DREAM}^{2} \mathrm{~S}$ : Deformable regions driven by an eulerian accurate minimization method for image and video segmentation. Int. J. Comput. Vision, 53:45-70, 2003.

[12] M. Kass, A. Witkin, and D. Terzopoulos. Snakes: Active contour models. Int. J. Comput. Vision, 1:321-332, 1988.

[13] M. E. Leventon, W. E. Grimson, and O. Faugeras. Statistical shape influence in geodesic active contours. In CVPR, 2000.

[14] N. Paragios and R. Deriche. Geodesic active regions for motion estimation and tracking. In ICCV, 1999 .
[15] C. Schnörr. Computation of discontinuous optical flow by domain decomposition and shape optimization. Int. J. Comput. Vision, 8:153-165, 1992.

[16] J. Sokolowski and J.-P. Zolésio. Introduction to shape optimization: Shape sensitivity analysis. Springer, 1992.

[17] A. Tsai, Jr. A. Yezzi, W. Wells, C. Tempany, D. Tucker, A. Fan, W. E. Grimson, and A. Willsky. A shape-based approach to the segmentation of medical imagery using level sets. IEEE Trans. Med. Imaging, 22:137-154, 2003.

[18] Y. Wang and L. H. Staib. Boundary finding with correspondence using statistical shape models. In CVPR, 1998.

[19] A. J. Yezzi, A. Tsai, and A. Willsky. A statistical approach to snakes for bimodal and trimodal imagery. In $I C C V, 1999$. 